\title{
Structure and rheology of binary mixtures in shear flow
}

\author{
F. Corberi \\ Istituto Nazionale per la Fisica della Materia, Unità di Salerno and Dipartimento di Fisica, Universitá di Salerno, 84081 \\ Baronissi (Salerno), Italy. \\ G. Gonnella and A. Lamura \\ Istituto Nazionale per la Fisica della Materia, Unità di Bari and Dipartimento di Fisica, Università di Bari, and Istituto \\ Nazionale di Fisica Nucleare, Sezione di Bari, via Amendola 173, 70126 Bari, Italy.
}

(October 30, 2018)

\begin{abstract}
Results are presented for the phase separation process of a binary mixture subject to an uniform shear flow quenched from a disordered to a homogeneous ordered phase. The kinetics of the process is described in the context of the time-dependent Ginzburg-Landau equation with an external velocity term. The large- $n$ approximation is used to study the evolution of the model in the presence of a stationary flow and in the case of an oscillating shear.

For stationary flow we show that the structure factor obeys a generalized dynamical scaling. The domains grow with different typical lengthscales $R_{x}$ and $R_{\perp}$ respectively in the flow direction and perpendicularly to it. In the scaling regime $R_{\perp} \sim t^{\alpha_{\perp}}$ and $R_{x} \sim \gamma t^{\alpha_{x}}$ (with logarithmic corrections), $\gamma$ being the shear rate, with $\alpha_{x}=5 / 4$ and $\alpha_{\perp}=1 / 4$. The excess viscosity $\Delta \eta$ after reaching a maximum relaxes to zero as $\gamma^{-2} t^{-3 / 2} . \Delta \eta$ and other observables exhibit log-time periodic oscillations which can be interpreted as due to a growth mechanism where stretching and break-up of domains cyclically occur.

In the case of an oscillating shear a cross-over phenomenon is observed: Initially the evolution is characterized by the same growth exponents as for a stationary flow. For longer times the phase separating structure cannot align with the oscillating drift and a different regime is entered with an isotropic growth and the same exponents of the case without shear.
\end{abstract}

47.20.Hw; 05.70.Ln; 83.50.Ax

\section{INTRODUCTION}

The kinetics of phase separation of a disordered system quenched into a multiphase coexistence region has been extensively studied in the last years [1]. The main features of the process are well understood: After an early stage during which ordered domains of the equilibrium phases are formed the segregation proceeds in the late stage by coarsening of ordered regions according to the power law growth $R(t) \sim t^{\alpha}$ for the average domains size. In binary liquids, the existence of several regimes characterized by different exponents $\alpha$, due to the presence of various growth mechanisms, is well established [2]. In these regimes the pair correlation function $C(r, t)$ verifies a dynamical scaling law according to which it can be written as $C(r, t) \simeq f(r / R)$, where $f(x)$ is a scaling function [3].

¿From the theoretical point of view the most relevant progresses have been achieved in the framework of the continuous approach based on the Cahn-Hilliard equation with a Ginzburg-Landau free energy functional, the time dependent Ginzburg-Landau (TDGL) model. Within this approach, which neglects hydrodynamics, the properties of the phase-separation kinetics can be efficiently studied by means of numerical simulations or analytically in the context of approximate theories, among which the so-called large- $n$ limit (one-loop approximation). For a vectorial system with an infinite number of components $n$, indeed, the TDGL model is exactly soluble. The one-loop approximation is known to provide a mean-field picture of the phase-separation process which captures the essence of the phenomenon at a semi-quantitative level 国.

In this paper we study the process of phase separation in a binary mixture subject to an uniform shear flow. When shear is applied to the system the time evolution is substantially different from that of ordinary spinodal decomposition. We consider both a stationary flow and an oscillating shear.

A stationary flow induces strong deformations of the domains formed after the quench [5] anisotropic and stretched along the flow direction. Consequently the growth rate along the flow is larger than in the other directions. In some experiments a power law increase of the typical size of the domains is observed and a value $\Delta \alpha=\alpha_{x}-\alpha_{\perp}$ in the range $0.8 \div 1$ for the difference between the exponents in the flow and in the shear directions is reported [8,9]. Two dimensional molecular dynamic simulations find a slightly smaller value [10]. In other experimental realizations, when the shear is strong enough, stringlike domains have been observed to extend 
macroscopically in the direction of the flow 11 preventing complete phase separation. In general, the scaling behavior of sheared systems is not clearly understood and the very existence of a scaling regime in different experimental systems is questionable.

In a previous paper 12] we have shown that the numerical solution of the one-loop approximation to the TDGL model for phase separation under shear exhibits a generalized scaling symmetry characterized by $\Delta \alpha=1$. In the scaling regime the structure factor and other observables exhibit the interesting feature of an oscillatory pattern which can be related to a mechanism of storing and dissipation of elastic energy where domains are stretched and broken cyclically. This new effect has been shown to persist up to the longest available time of our computation and represents the hallmark of a complex dynamical pattern induced by the presence of the shear. In a recent paper Rapapa and Bray [13], by solving asymptotically the one-loop equations, confirmed analytically the existence of a (multi)-scaling symmetry; in the long time limit, however, they do not recover the cyclical pattern described insofar and they infer 'that the observed oscillations are slowly-decaying preasymptotic transients'. Since their solution is obtained in the infinite time limit, then, a reference theory for the description of this remarkable phenomenon is lacking.

Given that the one-loop approximation is a mean-field solution in spirit the natural question of its accuracy for the description of the original model arises. A numerical analysis of the exact TDGL model has been performed recently in 14 where it is shown that the global picture of the one-loop approximation is adequate. In particular the oscillatory pattern is recovered. The existence of a scaling symmetry and the determination of the related exponents, however, has not been clearly established numerically mainly due to finite size effects limitations. The actual value of the growth exponents can be inferred by scaling [13] or renormalization group [14] arguments to be $\alpha_{\perp}=1 / 3$, as in the case without shear (we stress the fact that hydrodynamic effects are neglected in this model), and $\alpha_{x}=4 / 3$.

The shear also induces a peculiar rheological behavior. The break-up of the stretched domains liberates an energy which gives rise to an increase $\Delta \eta$ of the viscosity [15,16]. Experiments and simulations show that the excess viscosity $\Delta \eta$ reaches a maximum at $t=t_{m}$ and then relaxes to smaller values. The maximum of the excess viscosity is expected to occur at a fixed $\gamma t$ and to scale as $\Delta \eta\left(t_{m}\right) \sim \gamma^{-\nu}$ [6.8]. Simple scaling arguments predict $\nu=2 / 3$ [6], but different values have been reported [8]. All these features are adequately described by the TDGL already at the one-loop level.

In this paper we present a complete scenario of the behavior of the TDGL model for phase separation in a shear flow in the framework of the large- $n$ approximation. The behavior of the system is studied along the whole time history, from the instant of the quench onward, both in the presence of a steady flow and in the case of an oscillating shear where interesting effects are undercovered. Results are presented for two and three dimensional systems.

This paper is schematically divided as follows: In Sec.2 we specify the model and introduce the one-loop approximation that will be studied thoroughly in the following Sections. Section 3 is devoted to the analysis of the behavior of the model subjected to a steady flow. In Sec.4 the dynamics in the presence of an oscillatory shear is considered. In Sec. 5 we present a discussion of the results, debate some open problems and draw our conclusions.

\section{THE MODEL}

The binary mixture is described at equilibrium by a Ginzburg-Landau free-energy

$$
\mathcal{F}\{\varphi\}=\int d^{d} r\left\{\frac{a}{2} \varphi^{2}+\frac{b}{4} \varphi^{4}+\frac{\kappa}{2}|\nabla \varphi|^{2}\right\}
$$

where $\varphi$ is the order parameter which represents the concentration difference between the two components. The values of $b, \kappa$ are positive for any temperature $T$ of the fluid. The parameter $a$ separates stable states of the blend with $a>a_{c}(T)\left(a_{c}(T) \leq 0\right)$, from the thermodynamically unstable states with $a<a_{c}(T)$ where the system phase separates. The time evolution of the order parameter is given by the convection-diffusion equation

$$
\frac{\partial \varphi}{\partial t}+\vec{\nabla} \cdot(\varphi \vec{v})=\Gamma \nabla^{2} \frac{\delta \mathcal{F}}{\delta \varphi}+\eta
$$

where the gaussian stochastic field $\eta$, with expectations

$$
\begin{aligned}
\langle\eta(\vec{r}, t)\rangle & =0 \\
\left\langle\eta(\vec{r}, t) \eta\left(\vec{r}^{\prime}, t^{\prime}\right)\right\rangle & =-2 T \Gamma \nabla^{2} \delta\left(\vec{r}-\vec{r}^{\prime}\right) \delta\left(t-t^{\prime}\right)
\end{aligned}
$$

describes thermal fluctuations [5]. In Eq. (2) $\Gamma$ is a transport coefficient and the symbol $\langle\ldots\rangle$ indicates the ensemble average. The external velocity field here considered is of the form

$$
\vec{v}=\gamma y \vec{e}_{x}
$$


where $\gamma$ is the spatially homogeneous shear rate [ [] , that may however depend on time, and $\vec{e}_{x}$ is a unit vector in the flow direction. In the following we will consider a quench from an uncorrelated isotropic high temperature initial condition at the critical composition, i.e. with $\langle\varphi(\vec{r}, 0)\rangle=0$ and $\left\langle\varphi(\vec{r}, 0) \varphi\left(\vec{r}^{\prime}, 0\right)\right\rangle=\Delta \delta\left(\vec{r}-\vec{r}^{\prime}\right)$. The main observable for the description of the phase-separation kinetics is the structure factor

$$
C(\vec{k}, t)=<\varphi(\vec{k}, t) \varphi(-\vec{k}, t)>
$$

where $\varphi(\vec{k}, t)$ is the Fourier transform of the field $\varphi(\vec{r}, t)$ solution of Eq. (2) $)$. In the high temperature initial state we consider one has $C(\vec{k}, 0)=\Delta$.

The cubic term in the derivative $\delta \mathcal{F} / \delta \varphi$ prevents an exact solution of the Eq. (2), as in the case without shear [2]. However a soluble model is recovered in the one-loop approximation which amounts to the factorization of the cubic term of Eq. (2) as

$$
\varphi^{3} \rightarrow\left\langle\varphi^{2}\right\rangle \varphi
$$

It is possible to show [17] that the substitution (6) becomes exact in models with a vectorial order parameter when the number $n$ of its components becomes infinite. Since $\left\langle\varphi^{2}\right\rangle=S(t)$ does not depend on space, due to translational invariance, the substitution (6) formally linearizes the theory. The large- $n$ limit is a well developed approximation scheme in statistical mechanics which have been applied to different contexts [18]: Its validity and limitations are nowadays rather well understood [19].

In the large- $n$ approximation the dynamical equation for $C(\vec{k}, t)$ is:

$$
\frac{\partial C(\vec{k}, t)}{\partial t}-\gamma k_{x} \frac{\partial C(\vec{k}, t)}{\partial k_{y}}=-k^{2}\left[k^{2}+S(t)-1\right] C(\vec{k}, t)+k^{2} T
$$

where the function $S(t)$ is self-consistently given by

$$
S(t)=\int_{|\vec{k}|<q} \frac{d \vec{k}}{(2 \pi)^{d}} C(\vec{k}, t)
$$

and $q$ is a high momentum phenomenological cut-off. Notice that in Eq. (7) the parameters of the free-energy (11) and the mobility $\Gamma$ have been eliminated by a redefinition of the time, space and field scales. The rheological properties of the mixture are described in terms of the shear stress

$$
\sigma_{x y}(t)=-\int_{|\vec{k}|<q} \frac{d \vec{k}}{(2 \pi)^{d}} k_{x} k_{y} C(\vec{k}, t)
$$

and of the first and second normal stress differences

$$
\Delta N_{1}=\int_{|\vec{k}|<q} \frac{d \vec{k}}{(2 \pi)^{d}}\left[k_{y}^{2}-k_{x}^{2}\right] C(\vec{k}, t)
$$

and

$$
\Delta N_{2}=\int_{|\vec{k}|<q} \frac{d \vec{k}}{(2 \pi)^{d}}\left[k_{z}^{2}-k_{y}^{2}\right] C(\vec{k}, t) .
$$

For vectorial systems with $n>d$ ( $d$ is the spatial dimensionality) topological defects are not stable [2]. For large $n$, therefore, domains of the equilibrium phases are, strictly speaking, absent. Nevertheless, since from the solution of the one-loop equations presented below it is possible to identify characteristic growing lengths $R_{x}(t)$ and $R_{\perp}(t)$ in the flow and in the other directions it is natural to interpret these quantities as the trace of the domains size after the one-loop approximation procedure has been performed. In the following we will always use the word domains in this broad acception.

\section{STEADY SHEAR}

In this section we consider the case of a constant shear rate $\gamma$. Eq. (7) can be formally integrated, yielding 


$$
C(\vec{k}, t)=\Delta e^{-\int_{0}^{t} \mathcal{K}^{2}(u)\left[\mathcal{K}^{2}(u)+S(t-u)-1\right] d u}+2 T \int_{0}^{t} \mathcal{K}^{2}(u) e^{-\int_{0}^{u} \mathcal{K}^{2}(s)\left[\mathcal{K}^{2}(s)+S(t-s)-1\right] d s} d u
$$

where

$$
\overrightarrow{\mathcal{K}}(u)=\vec{k}+\gamma k_{x} u \vec{e}_{y}
$$

and $\vec{e}_{y}$ is the unit vector in the shear direction normal to the flow. For steady flow it is usual to define the excess viscosity as

$$
\Delta \eta(t)=\frac{\sigma_{x y}(t)}{\gamma}
$$

\section{A. Analytic solution in the short and long time limit}

The consistency condition (8) cannot be worked out along the whole time history of the system. For this reason in the following Sections the model Equations will be solved numerically both in $d=2$ and $d=3$. However the model can be solved in the short and long time limit.

\section{Short times}

For short times the linearized theory developed originally by Cahn and Hilliard [20] for the situation with $\gamma=0$ can be extended to the present case. This amounts to neglecting the quartic term in the local part of the free energy (11) since in the initial high temperature state the order parameter is small. With this approximation the solution of Eq. (2) reads

$$
C(\vec{k}, t)=\Delta e^{-\int_{0}^{t} \mathcal{K}^{2}(z)\left[\mathcal{K}^{2}(z)-1\right] d z}+2 T \int_{0}^{t} \mathcal{K}^{2}(z) e^{-\int_{0}^{z} \mathcal{K}^{2}(s)\left[\mathcal{K}^{2}(s)-1\right] d s} d z
$$

This approach applies to the original model and to the large- $n$ approximation as well because non-linear terms are neglected. It is well known that the linear theory describes the very initial transient of the phase-separation process, when domains are still forming. In this time domain the behaviour of the system in the presence of the flow is more interesting than in the simple case of an immobile fluid. A plot of the structure factor (15) is presented for a twodimensional system in Fig. (11) for the case $\gamma=1$ and $T=0$. Initially, when domains are forming but the shear flow has not yet produced sensible effects, the structure factor evolves assuming the typical structure of a circular volcano, similarly to what happens in the case without shear. At $\gamma t \simeq 0.5$ the anisotropy induced by the shear produces a deformation in the profile of the edge of the volcano from a ring-like geometry into an ellipse, whose major axis forms with the positive direction of the $k_{y}$ axis an angle of approximatively $45^{\circ}$ (see Fig. (1)). At the same time small dips start to develop in the edge at the ends of the axes of the ellipse and four peaks can be clearly observed at $\gamma t \simeq 2$. As time goes by, the angle formed by the major axis of the ellipse with the $k_{y}$-direction decreases and the dips in the profile of $C(\vec{k}, t)$ along the major axis develop until $C(\vec{k}, t)$ almost consists of two separated foils, at $\gamma t \simeq 4$, when a couple of peaks prevails. The same initial pattern is also observed [14 by numerically solving the full model equation (2). At later times, however, the presence of the non-linear terms becomes fundamental and the linear theory breaks down, as in the case without shear. It is important to stress the fact that the presence of four peaks in the structure factor is exhibited already at the linear theory level of approximation. We will see in the following sections that the very existence of a multiply peaked $C(\vec{k}, t)$ produces a rich dynamical pattern originating an oscillatory phenomenon.

\section{Long times}

The self-consistency condition (8) has been worked out explicitly in the long-time domain in [13]. It is found that

the model is interested by a multiscaling symmetry, as in the case without shear 21], characterized by the growth of the characteristic lengthscales as 


$$
R_{x} \sim \gamma\left(\frac{t^{5}}{\ln t}\right)^{\frac{1}{4}}
$$

and

$$
R_{\perp} \sim\left(\frac{t}{\ln t}\right)^{\frac{1}{4}}
$$

in the directions of the flow and perpendicular to it respectively. The excess viscosity and the normal stress differences behave as

$$
\begin{gathered}
\Delta \eta(t) \sim \gamma^{-2}\left(\frac{\ln t}{t^{3}}\right)^{\frac{1}{2}} \\
\Delta N_{1} \sim \Delta N_{2} \sim\left(\frac{\ln t}{t}\right)^{\frac{1}{2}}
\end{gathered}
$$

The same behaviors (apart from logarithmic corrections) is obtained in 12 by means of a scaling ansatz.

\section{B. Numerical solution}

We present in this Section the results of the numerical integration of the large- $n$ equation (7) which allows to follow the whole time history of the phase-separation process. We restrict ourselves to the case with $T=0$. An Euler first order discretization scheme has been implemented in $d=2$ and $d=3$ on $d$-dimensional lattices with 201 mesh points per each direction. For long times the structure factor is strongly peaked around typical wavevectors which move towards zero as time goes on (see Fig 2). Given that the support of $C(\vec{k}, t)$ also shrinks to zero it is possible to greatly improve the quality of the numerical computation by using a self-adaptive mesh algorithm that follows the evolution of the support of the structure factor. We have solved Eq. (7) for various values of the shear rate $\gamma$ in the range $\left[10^{-4}, 10^{-2}\right]$. We found that the qualitative behavior is the same for all the values of $\gamma$ considered. ¿From the knowledge of the structure factor we compute the characteristic lengths $R(t)$ as

$$
R_{x}(t)=\frac{1}{\sqrt{\overline{k_{x}^{2}}}}
$$

where

$$
\overline{k_{x}^{2}}=\frac{\int d \vec{k} k_{x}^{2} C(\vec{k}, t)}{\int d \vec{k} C(\vec{k}, t)}
$$

and the same for the other directions.

$$
d=2
$$

The behavior of $C(\vec{k}, t)$ is shown in Fig. (2) for $\gamma=0.001$. Initially the evolution of the structure factor is resemblant to the one observed in Fig. (11) where the linear theory for $C(\vec{k}, t)$ was plotted. Later on, however, the linear theory fails because the non-linearities become relevant, and the long-time regime is entered. This is characterized by the shrinking of the support of $C(\vec{k}, t)$ towards the origin with different rates for the shear and the flow directions so that the tilt angle, namely the direction along which $C$ is aligned, decreases in time. The structure factor is divided into two separated foils which are symmetric due to the property $C(\vec{k}, t)=C(-\vec{k}, t)$. In each foil two distinct peaks can be observed located at $\left(k_{x_{1}}, k_{y_{1}}\right)$ and $\left(k_{x_{2}}, k_{y_{2}}\right)$ with $\left|k_{x_{1}}\right| \simeq 2\left|k_{x_{2}}\right|$ and $\left|k_{y_{1}}\right| \simeq 2\left|k_{y_{2}}\right|$. Their heights change in time. The first peak to prevail is that located at $\left(k_{x_{1}}, k_{y_{1}}\right)$, while the other peak dominates later. As time elapses the two peaks are observed to prevail alternatively. This oscillatory behavior continues up to the longest times of our computations.

In Fig. (3) the quantities $(\gamma \ln t)^{1 / 4} R_{x}(t)$ and $(\gamma \ln t)^{1 / 4} R_{y}(t)$ are plotted against the strain $\gamma t$. According to Eqs.(16, 17) for long times these quantities should collapse, for different values of the shear, on two power-law mastercurves with exponents $5 / 4$ and $1 / 4$, respectively. Here we observe that the collapse is indeed good, but the predicted 
power-law behavior is modulated by an oscillatory pattern. These oscillations are observed to be periodic on a log-time axis and persist up to the limit of the computational time.

We now consider the rheological behavior of the mixture by plotting in Fig. (伍) the quantity $(\gamma / \ln t)^{1 / 2} \Delta \eta(t)$ against the strain. This quantity reaches a maximum at $\gamma t \simeq 3.5$ and then decrease as also found in experiments [B]. For long times Eq. (18) would predict a data collapse for different $\gamma$ on a single power-law master-curve with exponent $-3 / 2$. Here the situation is similar to the previous figure, in that the predicted behavior is modulated by log-time periodic oscillations. On the bases of simple scaling arguments the maximum of the excess viscosity $\Delta \eta\left(t_{m}\right)$ is expected to occur at a fixed $\gamma t$ and to scale as $\Delta \eta\left(t_{m}\right) \sim \gamma^{-\nu}$, with $\nu=2 / 3$ [6, 8]. These arguments do not directly apply to the one-loop approximation since, due to the mean field nature, the exponents are different. The asymptotic solution (18) is not adequate to this early stage effect. The $\gamma$ dependence of $\Delta \eta\left(t_{m}\right)$ is plotted in the inset of Fig. 目 showing that a power-law behaviour with $\nu \simeq 0.6$ is obeyed, in partial agreement with the aforementioned scaling arguments.

In Fig. (5) we report the numerical results for the first normal stress by plotting $(\gamma \ln t)^{-1 / 2} \Delta N_{1}$ against $\gamma t$ with $\gamma=0.01$. We find that $\Delta N_{1}(t)$ scales asymptotically as predicted by Eq. (19) again modulated by an oscillatory pattern.

The periodic oscillations observed in all the physical observables are due to the competition between the different peaks of $C(\vec{k}, t)$. Let's refer to the behavior of the excess viscosity to understand how this competition affects the rheological quantities, using the features of the structure factor to obtain information about the domains evolution under the action of shear. $\Delta \eta$ reaches its first maximum when the shape of $C(\vec{k}, t)$ is such that the peak located at $\left(k_{x_{1}}, k_{y_{1}}\right)$ prevails and the difference between the height of the two peaks is maximal. At this time the domains are elongated by the flow and there is a prevalence of thin domains in the system. As these string-like domains are stretched further, they eventually break up into two or more domains, dissipating the stored energy. This has two effects: the excess viscosity decreases and, on the other hand, the thick domains, which have not yet been broken, prevail. In this situation the other peak of $C(\vec{k}, t)$ (which is located at $\left(k_{x_{2}}, k_{y_{2}}\right)$ and represents the smaller features) grows faster until it prevails and $\Delta \eta$ reaches a minimum. This behavior is reproduced with a characteristic frequency in log-time. Recently, a similar behavior has been observed in the numerical simulation of the full model Equation (2) [14.

$$
d=3
$$

In this section we report the result of the numerical solution of Eq. (7) in $d=3$. In Fig. (6) the time evolution of the structure factor in the special planes $k_{x}=0, k_{y}=0$ and $k_{z}=0$ is shown for $\gamma=0.001$. In the plane $k_{z}=0 C(\vec{k}, t)$ behaves analogously to the previously discussed two dimensional case. The structure factor on the plane $k_{y}=0$ gives information relative to the observation of the system along the shear direction: No velocity gradient is present in the plane perpendicular to this orientation, but there are different velocities in the $x$ and $z$ directions. This allows to explain the observed behavior which is rather different from the one observed at $k_{z}=0$. The structure factor develops initially a circular volcano, as without shear. The edge of the volcano is progressively deformed by the shear into an ellipse with the major axis along the $k_{z}$ direction. The dips in the edge of the volcano at values of $k_{x} \simeq 0$ develop with time so that at $\gamma t \simeq 1, C(\vec{k}, t)$ is made of two foils but these are not completely separated. During the time evolution the axes of the ellipse shrink; the decrease is faster along the $k_{x}$ direction. The two foils are never completely separated and the angle formed with the $k_{z}$ direction is zero, as observed in experiments [9]. At $\gamma t \simeq 5$ two well-formed peaks start to develop and grow on each foil of $C(\vec{k}, t)$. These four peaks have the same height and their relative heights do not change in time, as it can be seen at $\gamma t=20$ in the picture, differently from the situation on the $k_{z}=0$ plane.

In the $k_{x}=0$ plane the shear has no effect at all and the structure factor remains circular during its evolution.

The computed behavior of $R_{x}(t)$ and $R_{y}(t)$ is similar to that of the two-dimensional case. We also find $R_{z}(t) \sim R_{y}(t)$, as expected.

We report in Fig. (7) the plots of $(\gamma / \ln t)^{1 / 2} \Delta \eta(t),(\gamma \ln t)^{-1 / 2} \Delta N_{1}(t)$ and $-(\gamma \ln t)^{-1 / 2} \Delta N_{2}(t)$ as functions of $\gamma t$. It appears that the rheological quantities still have amplitudes which are modulated by log-time oscillations which are in phase among them. The origin of such oscillations has to be found again in the oscillations of the peaks of the structure factor in the plane $k_{z}=0$. Since the support of $C(\vec{k}, t)$ shrinks towards the origin faster in the $k_{z}$ than in the $k_{y}$ direction the second normal stress difference $\Delta N_{2}(t)$ is negative. This is in accordance with general experimental experience 22. 


\section{OSCILLATING SHEAR}

In this Section we consider the case of a time-dependent shear rate with

$$
\gamma(t)=\gamma_{o} \cos \omega t
$$

This situation is of great experimental relevance expecially for probing the viscoelastic properties of the phase separating binary mixture.

We solved the Eq. (7) numerically in $d=2$ using the same numerical scheme as in the case of steady shear, for different values of $\gamma_{0}$ and $\omega$. We will describe below the case $\gamma_{0}=10^{-3}, \tau=2 \pi / \omega=6 \times 10^{3}$. The time evolution of the structure factor in the first cycle of $\gamma(t)$ is shown in Fig. (8). The dynamical pattern is analogous to the one with $\gamma=$ const. for times $t<\tau / 4$ as it can be seen at $\gamma_{o} t=1.5$. Then the time dependent velocity field modifies the behaviour of the blend with respect to the case of a steady flow. In particular, at the end of the first oscillation, the four peaks of $C(\vec{k}, t)$ are located at comparable distances from the origin of the $k$-space differently from what observed in Fig. (2) at $\gamma t=6$. The two highest maxima at $\gamma_{0} t=6$ in Fig. (8) are characterized by $\left|k_{y}\right| \gg\left|k_{x}\right|$. During the later time evolution these peaks grow and move towards the origin. The position in the $k$-plane of the other peaks rotates back and forth cyclically along an approximatively circular path. The radius of this trajectory shrinks towards the origin at a rate comparable with that of the position of the other peaks. In the asymptotic regime the four peaks have approximatively the same height and the cyclical rotation of the peak position persists. This is shown in Fig. (9) where the configurations of the structure factor are shown at each quarter of oscillation of the shear rate in the asymptotic stage.

In Fig. (10) the evolution of the characteristic lengths $R_{x}(t), R_{y}(t)$ is plotted against $\gamma_{0} t$. We also plot, in the inset, the time average of these quantities over a period $\tau$, in order to smoothen out the superimposed oscillations. Here we observe, for times $t<\tau$, growth laws analogous to the steady shear case, namely $R_{x}(t) \sim t^{5 / 4}$ and $R_{y}(t) \sim t^{1 / 4}$. The growth exponent of $R_{x}$ changes smoothly, from $t \simeq \tau$ onward, from $5 / 4$ to the asymptotic value $1 / 4$ which is reached at $\gamma_{o} t \sim 80$ when all the four peaks of the structure factor have the same height. The gradual crossover of $\alpha_{x}$ from $5 / 4$ to $1 / 4$ can be better observed for larger values of $\tau$, since the regime with $\alpha_{x}=5 / 4$ persists for a longer time. This is shown in Fig. (11), where the evolution of $R_{x}(t), R_{y}(t)$ is plotted against $\gamma_{0} t$ for $\tau=5 \times 10^{5}$. For small $\tau$, instead, $R_{x}$ and $R_{y}$ grow with the same exponent $1 / 4$ from the beginning.

These observations suggest the following physical interpretation: for $t<\tau / 2$, since $\gamma$ does not change sign, the evolution of the blend is comparable to the case with a constant shear rate. In particular, if $\tau$ is sufficiently large to exceed the initial stage when domains are forming, the power growth laws described in Sec. 3 for $R_{x}, R_{\perp}$ are observed with $\alpha_{x}=5 / 4$ and $\alpha_{\perp}=1 / 4$. On timescales much longer then $\tau$, however, the network of the larger domains cannot be efficiently tilted along the flow orientation which changes periodically its sign. This is confirmed by the behaviour of the two peaks with $\left|k_{y}\right| \gg\left|k_{x}\right|$ whose position in the $k$-plane moves toward the origin but does not cross the $k_{x}=0$ plane, as it would be the case if the orientation of the domains corresponding to these peaks were reversed. In this situation the difference $\Delta \alpha=1$ between the exponents in the flow and shear direction cannot be sustained, because the larger domains are not directed along the flow orientation at all times, and a growth law with the same exponent $1 / 4$ in all the directions is obeyed. It is interesting to notice that the other peaks, which represent smaller domains formed by the break-up of the larger ones, crosses the $k_{y}=0$ plane during their rotation every half period of $\gamma$. This suggests that these features are tilted by the oscillating shear and follow the flow orientation. Then we expect to observe in a real blend two type of domains which respond differently to the oscillations of the flow: a network of large and elongated structures which maintains the orientation imposed during the first half period of $\gamma$ and a multitude of more isotropic features, generated by the break-up of strained regions, which oscillate following the flow.

For studying rheological properties it is customary [23] to introduce a complex viscosity $\eta^{*} \equiv \eta^{\prime}-i \eta^{\prime \prime}$ which is related to the shear stress by

$$
\sigma_{x y}(t)=\gamma_{o}\left(\eta^{\prime} \cos \omega t+\eta^{\prime \prime} \sin \omega t\right)
$$

when Eq. 22, holds. It is also useful to consider [24] another representation of the shear stress given by

$$
\sigma_{x y}(t)=C \sin (\omega t+\phi)
$$

The connection between Eqs. (23) and (24) is given by

$$
C=\gamma_{o} \sqrt{\eta^{\prime 2}+\eta^{\prime 2}}
$$

and 


$$
\tan \phi=\frac{\eta^{\prime}}{\eta^{\prime \prime}} .
$$

By defining $\gamma^{*}(t)=\gamma_{o} e^{i \omega t}$, we can write Eq. (23) as

$$
\sigma_{x y}(t)=\operatorname{Re}\left[\eta^{*} \gamma^{*}(t)\right]
$$

In order to relate the real and imaginary parts of the viscosity to physical quantities Eq. (23) can be casted as

$$
\sigma_{x y}(t)=\eta \gamma(t)+G \int_{0}^{t} \gamma\left(t^{\prime}\right) d t^{\prime}
$$

where $\eta=\eta^{\prime}, G=\omega \eta^{\prime \prime}$ and the identity $\sin \omega t=\omega \int_{0}^{t} \cos \omega t^{\prime} d t^{\prime}$ has been used.

The coefficient $\eta$ in the r.h.s. of the Eq. (28) multiplies the portion of the shear stress in phase with the shear rate and represents the viscosity of a viscoelastic fluid. The integral of the second term of the r.h.s. of the Eq. (28) can be identified with the shear strain present in the mixture at time $t$. The coefficient $G$ is therefore the effective elastic shear modulus of the fluid. Pure viscous behavior corresponds to $G=0(\phi=\pi / 2)$, pure elastic behavior to $\eta=0$ $(\phi=0)$ 24].

In order to compute $\eta$ and $G$ during the phase separation we calculated by numerical integration the shear stress using its general definition (9). By writing

$$
\sigma_{x y}(t)=A \cos \omega t+B \sin \omega t
$$

it follows that $\eta=A(t) / \gamma_{o}$ and $G=\omega B(t) / \gamma_{o}$. In general, $A$ and $B$ depend on time. During a single shear oscillation, however, we expect that the Eq. (29) holds as a good approximation with constant values for $A$ and $B$. In this way $\sigma_{x y}(t)$ is expressed in terms of the first two coefficients in a Fourier series expansion over the interval of scaled time of duration $2 \pi$. The values we obtain may be referred to the time that locates the middle of the interval. Thus we get

$$
\begin{aligned}
\eta\left(\left(m-\frac{1}{2}\right) \tau\right) & =\frac{1}{\gamma_{o} \pi} \int_{(m-1) \cdot 2 \pi}^{m \cdot 2 \pi} \sigma_{x y}(t / \omega) \cos t d t \\
G\left(\left(m-\frac{1}{2}\right) \tau\right) & =\frac{\omega}{\gamma_{o} \pi} \int_{(m-1) \cdot 2 \pi}^{m \cdot 2 \pi} \sigma_{x y}(t / \omega) \sin t d t
\end{aligned}
$$

where $m=1,2, \ldots$

In Fig. (12) we report the plots of $\eta$ and $G$ against $\gamma_{o} t$. The viscosity shows a crossover between a power law decay with exponent $-3 / 2$ at short times and an asymptotic behavior whose exponent is $-1 / 2$. This can be explained observing that, for the steady shear case, the dynamic viscosity $\eta$ coincides with the excess viscosity which scales with the inverse of the domains volume $V$. When an oscillatory shear is applied $V$ crosses over from an initial power law increase $V \sim t^{3 / 2}$, similar to the one for the case with steady shear (see Fig. (3)), to a slower growth $V \sim t^{1 / 2}$, as already discussed above for $R_{x}(t)$, producing a corresponding crossover in $\eta$.

¿From the computed values of $\eta$ and $G$ we estimated the phase angle $\phi$, which, according to Eqs. (26) and (28), is given by $\phi=\arctan \left(\frac{\eta}{G} \omega\right)$. In Fig. (13) we report the time evolution of $\phi$ as a function of $\gamma_{o} t$. It can be seen that $\phi$ decreases with time to reach an asymptotic value which is approximately 0.016. Accordingly, the system we are investigating shows in the asymptotic stage a behavior which is essentially elastic. The experimental data of [25] confirm this behavior.

\section{SUMMARY AND DISCUSSION}

In this paper we have studied the kinetics of a phase-separating binary fluid, in the presence of a shear flow, by means of the TDGL model. It is nowadays well established that the corresponding model with $\gamma=0$ accurately describes the main features of the segregation process in binary alloys, where hydrodynamics can be neglected. In viscous fluids, such as polymeric blends, the validity of the present approach is limited to the early stage of spinodal decomposition; for longer times one should consider the full hydrodynamic description [2].

When the shear is applied to a fluid the behavior of the system is profoundly changed under many respects and a general agreement on the predictivness of the proposed models is matter of general debate. A discussion on possible effects of hydrodynamics is presented in [3]. Moreover, the numerical solution of the TDGL model with shear poses 
serious problems due to discretization limitations and finite size effects and, although some progresses have been recently achieved [14], a satisfactory description is nowadays not available. In this scenario it is important to devise a simple analytical scheme providing the fundamental tools for the comprehension of the fluid dynamics. A natural choice in the field of growth kinetics is the large- $n$ approximation, that has been thoroughly studied in the case without shear, where it has proven to give a reliable description of the segregation process, although at a mean field level.

In this paper the behavior of the TDGL model in the one-loop approximation is studied in detail, and the whole time evolution of the blend is considered, from the quenching instant onward; the cases of a stationary flow and of an oscillating shear have been examined. In doing so we undercover a very rich dynamical pattern, where not only some experimental findings are reproduced, but new predictions are allowed. After an early stage, which is accurately described by the linear theory á la Cahn-Hilliard, the presence of the velocity field produces an anisotropic power-law growth of the characteristic lengths $R_{x}, R_{\perp}$ respectively in the flow direction and perpendicularly to it. The value of the exponent $\alpha_{\perp}=1 / 4$ in the directions perpendicular to the flow is the same as in models with vectorial conserved order parameter without shear; although the actual value of this exponent is not expected to be accurate for real fluids (since, even without shear, the exponent obtained at the same level of approximation is known to corresponds to the Lifshitz-Slyozov exponent $\alpha=1 / 3$ for scalar fields) a growth exponent $\alpha_{\perp}$ unaffected by the presence of shear has been obtained also by scaling [13] and renormalization group [14 arguments applied to the full model equations and is also measured in experiments [8]. Moreover a difference $\Delta \alpha=1$ between the flow and shear exponents is also expected to be obtained by releasing the present approximation [13, 14 and is observed in some experiments. In the case of a stationary flow the anisotropic growth governed by these exponents is observed from the onset of the scaling regime onwards. The power law behavior of any observable is decorated by log-time periodic oscillations. These oscillations characterize the scaling regime up to the longest simulated time but they are not observed in the asymptotic solution presented in [13]. Given that log-time periodicity appears to be a rather common feature being observed, besides segregating fluids, during fracturing of heterogeneous solids [26.27] and in stock market indices [28] for instance, it would then be interesting to devise an analytical approach to enlighten the origin of this new phenomenon at least in the present model.

In experiments with real fluid systems carried out by Laufer et al. [29] and, successively, by Mani et al. [30] and Migler et al. [31] a double overshoot in the time behavior of the viscosity and of the normal stress is observed and an interpretation in terms of break-up and recombination of the domains network is proposed. On the bases of our results it is plausible that this double overshoot represents the first part of a log-time periodic phenomenon which could be hopefully detected with a suitable experimental setup. In the model we have studied the oscillatory behaviour is due to the competition between the different maxima of a four-fold peaked structure factor. The presence of these maxima is interpreted is Sec. III B as due to the existence of different types of domains and the recurrent prevalence of each peak is suggested to be caused by the interplay between these kind of regions. A structure factor with four maxima has also been observed in polymer mixtures [31]; however to our knowledge the connection between the alternative dominance of the peaks of $C(\vec{k}, t)$ and the overshoots observed in the viscosity and in the stresses has never been discussed insofar, perhaps due to insufficient resolution, although an experimental confirmation of this hypotheses would be desirable.

When an oscillating shear is present, the anisotropic regime discussed so far for the steady shear case crosses over to an isotropic growth when domains are fully developed. In this late stage, from the analysis of the behavior of the structure factor, we conjecture again the existence of two types of domains responding differently to the flow oscillations: the network of elongated structures keeps the orientation assumed during its formation in the early stage while small features generated by scission of strained parts oscillate in phase with the flow. In this late stage the growth kinetics is regulated by the same exponents as without flow. We are not aware of experiments reporting these features: It would be interesting to devise an experimental set-up for testing this prediction.

\section{ACKNOWLEDGMENTS}

F.C. is grateful to M.Cirillo, R.Del Sole and M.Palummo for hospitality in the University of Rome. F.C. acknowledges support by the TMR network contract ERBFMRXCT980183 and by PRA-1999 INFM and MURST(PRIN $97)$. 
[1] K. Binder in "Phase Transitions in Materials", Materials Science and Technology Vol. 5, eds. R.W. Cahn, P. Haasen, and E.J. Kramer (VCH Weinheim 1990); H. Furukawa, Adv. in Phys. 34, 703 (1985); J. D. Gunton et al. , in "Phase Transitions and Critical Phenomena", Vol. 8, eds. C. Domb and J.L. Lebowitz (Academic 1983).

[2] A.J. Bray, Adv. in Phys. 43, 357 (1994).

[3] A recent review on the influence of hydrodynamic effects on scaling properties is presented in: J.M. Yeomans, Phase Ordering in Fluids, preprint OUTP-99-06S (1999). See, also, M.E. Cates, V.M. Kendon, P. Bladon, and J.C. Desplat, Faraday Discussions 112, 1 (1999).

[4] A. Coniglio, P. Ruggiero, and M. Zannetti, Phys. Rev. E 50, 1046, (1994).

[5] For a review, see A. Onuki, J. Phys.: Condens. Matter 9, 6119 (1997).

[6] T. Ohta, H. Nozaki, and M. Doi, Phys. Lett. A 145, 304 (1990); J. Chem. Phys. 93, 2664 (1990).

[7] D.H. Rothman, Europhys. Lett. 14, 337 (1991).

[8] J. Läuger, C. Laubner, and W. Gronski, Phys. Rev. Lett. 75, 3576 (1995).

[9] C.K. Chan, F. Perrot, and D. Beysens, Phys. Rev. A 43, 1826 (1991).

[10] P. Padilla and S. Toxvaerd, J. Chem. Phys. 106, 2342 (1997).

[11] T. Hashimoto, K. Matsuzaka, E. Moses, and A. Onuki, Phys. Rev. Lett. 74, 126 (1994).

[12] F. Corberi, G. Gonnella, and A. Lamura, Phys. Rev. Lett. 81, 3852 (1998).

[13] N.P. Rapapa and A.J. Bray, Phys. Rev. Lett. 83, 3856 (1999).

[14] F. Corberi, G. Gonnella, and A. Lamura, Phys. Rev. Lett. 83, 4057 (1999).

[15] A. Onuki, Phys. Rev. A 35, 5149 (1987).

[16] See, for instance: A.H. Krall, J.V. Sengers, and K. Hamano, Phys. Rev. Lett. 69, 1963 (1992).

[17] See, e.g., S.k. Ma in "Phase Transitions and Critical Phenomena", Vol. 6, eds. C. Domb and M.S. Green (Academic 1976)

[18] F.de Pasquale, G.F. Mazenko, P. Tartaglia, and M. Zannetti, Phys. Rev. B 37, 296 (1988); S.C Glotzer and A. Coniglio, Phys. Rev. E 50, 4241 (1994); U. Marini Bettolo Marconi and F. Corberi, Europhys. Lett. 30, 349 (1995).

[19] C. Castellano, F. Corberi, and M. Zannetti, Phys. Rev. E 56, 4973 (1997).

[20] J.W. Cahn and J.E. Hilliard, J. Chem. Phys. 28, 258 (1958); H.E. Cook, Acta Metall. 18, 297 (1970).

[21] Simple scaling is expected when the large-n approximation is released [A.J.Bray and K.Humayun, Phys. Rev. Lett. 68, 1559 (1992)].

[22] H. A. Barnes, J. F. Hutton, and K. Walters, "An Introduction to Rheology", (Elsevier 1989)

[23] See, e.g., A.H. Krall, J.V. Sengers, and K. Hamano, Phys. Rev. E 48, 357 (1993).

[24] G. Pätzold and K. Dawson, Phys. Rev. E 54, 1669 (1996).

[25] A.H. Krall, J.V. Sengers, and K. Hamano, Phys. Rev. Lett. 69, 1963 (1992).

[26] R.C. Ball and R. Blumenfeld, Phys. Rev. Lett. 65, 1784 (1990).

[27] M. Sahimi and S. Arbabi, Phys. Rev. Lett. 77, 3689 (1996); see also D. Sornette, Phys. Rep. 297,239 (1998).

[28] A. Johansen and D. Sornette, preprint in cond-mat/9907270.

[29] Z. Laufer, H.L. Jalink, and A.J. Staverman, Journal of Polymer science 11, 3005 (1973).

[30] S. Mani, M.F. Malone, H.H. Winter, J.L. Halary, and L. Monnerie, Macromolecules 24, 5451 (1991); S. Mani, M.F. Malone, and H.H. Winter, Macromolecules 25, 5671 (1992).

[31] K. Migler, C. Liu, and D.J. Pine, Macromolecules 29, 1422 (1996). 


\section{CAPTIONS}

FIG. 1. The evolution of the structure factor in the linear approximation for $\gamma=1$. The range of $k_{x}$ and $k_{y}$ varies from -2 to 2 . At $\gamma t=4$ the highest peaks are located at $\left(k_{x}, k_{y}\right) \simeq(0.0, \pm 0.75)$, the other two at $\left(k_{x}, k_{y}\right) \simeq(\mp 0.38, \pm 0.80)$.

FIG. 2. The evolution of the structure factor from the numerical solution of Eq. ( $($ ) in $d=2$ for $\gamma=0.001$ and $T=0$. The range of $k_{x}$ and $k_{y}$ varies, at increasing times, as $-0.6 \leq k_{x}, k_{y} \leq 0.6$ at $\gamma t=0.05,1 ;-0.15 \leq k_{x} \leq 0.15$ and $-0.6 \leq k_{y} \leq 0.6$ at $\gamma t=6,8 ;-0.075 \leq k_{x} \leq 0.075$ and $-0.6 \leq k_{y} \leq 0.6$ at $\gamma t=10 ;-0.01875 \leq k_{x} \leq 0.01875$ and $-0.3 \leq k_{y} \leq 0.3$ at $\gamma t=45$.

FIG. 3. Data collapse (scaling plot) for the domains radii in $d=2$. The quantities $(\gamma \ln t)^{1 / 4} R_{x}(t)$ and $(\gamma \ln t)^{1 / 4} R_{y}(t)$ are plotted against the strain $\gamma t$ for different values of the shear rate: $(\triangle) \gamma=0.0001,(\circ) \gamma=0.001,(\bullet) \gamma=0.01$. The two straight lines have slope $5 / 4$ and $1 / 4$.

FIG. 4. Data collapse (scaling plot) for the excess viscosity in $d=2$. The quantity $(\gamma / \ln t)^{1 / 2} \Delta \eta(t)$ is plotted against the strain $\gamma t$ for different values of the shear rate: $(\triangle) \gamma=0.0001,(\circ) \gamma=0.001,(\star) \gamma=0.005,(\bullet) \gamma=0.01$. The straight line has slope $-3 / 2$. The inset shows the maxima of $\Delta \eta$ as a function of $\gamma$. The slope of the straight line is 0.6 .

FIG. 5. The first normal stress $\Delta N_{1}$ multiplied by $(\gamma \ln t)^{-1 / 2}$ as a function of $\gamma t$. The value of $\gamma$ is 0.01 . The slope of the straight line is $-1 / 2$.

FIG. 6. The structure factor from the numerical solution of Eq. (目) in $d=3$ at consecutive times for $\gamma=0.001$ and $T=0$. From up to bottom the sections $k_{x}=0, k_{y}=0$ and $k_{z}=0$ of the structure factor are shown. The range of $k_{x}, k_{y}$ and $k_{z}$ varies as $-0.6 \leq k_{x}, k_{y}, k_{z} \leq 0.6$ at $\gamma t=1 ;-0.0375 \leq k_{x} \leq 0.0375,-0.3 \leq k_{y} \leq 0.3$ and $-0.3 \leq k_{z} \leq 0.3$ at $\gamma t=20$.

FIG. 7. $(\gamma / \ln t)^{1 / 2} \Delta \eta(t)(*),(\gamma \ln t)^{-1 / 2} \Delta N_{1}(t)(\circ)$ and $-(\gamma \ln t)^{-1 / 2} \Delta N_{2}(t)(\bullet)$ as functions of the strain $\gamma t$. The straight lines have slope $-3 / 2$ and $-1 / 2$.

FIG. 8. The structure factor at each quarter of the first $\gamma(t)$ oscillation. The range of $k_{x}$ and $k_{y}$ varies, at increasing times, as $-0.6 \leq k_{x}, k_{y} \leq 0.6$ at $\gamma t=1.5 ;-0.3 \leq k_{x} \leq 0.3$ and $-0.6 \leq k_{y} \leq 0.6$ at $\gamma t=3,4.5 ;-0.3 \leq k_{x}, k_{y} \leq 0.3$ at $\gamma t=6$.

FIG. 9. The structure factor at each quarter of a single oscillation in the asymptotic stage. The range of $k_{x}$ and $k_{y}$ is $-0.15 \leq k_{x}, k_{y} \leq 0.15$ at each time.

FIG. 10. The size of the domains in the $x$ (upper curve) and $y$ (lower curve) directions are plotted against $\gamma_{0} t$ for $\tau=6 \times 10^{3}$. In the inset the same quantities ( $*$ and $\bullet$ for the $x$ and $y$ direction, respectively) averaged over every single period of oscillation are plotted at times $(m-1 / 2) \gamma_{o} \tau$, with $m=1,2, .$. , against $\gamma_{0} t$. The two straight lines have slope $1 / 4$.

FIG. 11. The size of the domains in the $x$ (upper curve) and $y$ (lower curve) directions in the case $\tau=5 \times 10^{5}$. The two straight lines have slope $5 / 4$ and $1 / 4$.

FIG. 12. The viscosity $\eta(*)$ and the elastic shear modulus $G(\bullet)$ for $\tau=6 \times 10^{3}$ at times $(m-1 / 2) \gamma_{o} \tau$ with $m=1,2, \ldots$ The straight lines have slope $-3 / 2$ and $-1 / 2$.

FIG. 13. The time evolution of the phase angle $\phi$ for $\tau=6 \times 10^{3}$ at times $(m-1 / 2) \gamma_{o} \tau$ with $m=1,2, \ldots$ 
This figure "fig_1.gif" is available in "gif" format from: http://arxiv.org/ps/cond-mat/0001342v1 
This figure "fig_2.gif" is available in "gif" format from: http://arxiv.org/ps/cond-mat/0001342v1 
This figure "fig_3.gif" is available in "gif" format from: http://arxiv.org/ps/cond-mat/0001342v1 
This figure "fig_4.gif" is available in "gif" format from: http://arxiv.org/ps/cond-mat/0001342v1 
This figure "fig_5.gif" is available in "gif" format from: http://arxiv.org/ps/cond-mat/0001342v1 
This figure "fig_6.gif" is available in "gif" format from: http://arxiv.org/ps/cond-mat/0001342v1 
This figure "fig_7.gif" is available in "gif" format from: http://arxiv.org/ps/cond-mat/0001342v1 
This figure "fig_8.gif" is available in "gif" format from: http://arxiv.org/ps/cond-mat/0001342v1 
This figure "fig_9.gif" is available in "gif" format from: http://arxiv.org/ps/cond-mat/0001342v1 
This figure "fig_10.gif" is available in "gif" format from: http://arxiv.org/ps/cond-mat/0001342v1 
This figure "fig_11.gif" is available in "gif" format from: http://arxiv.org/ps/cond-mat/0001342v1 
This figure "fig_12.gif" is available in "gif" format from: http://arxiv.org/ps/cond-mat/0001342v1 
This figure "fig_13.gif" is available in "gif" format from: http://arxiv.org/ps/cond-mat/0001342v1 